\title{
Investigation of the influence of excess free volume on the mobility of triple junctions of grain boundaries
}

\author{
D. V. Novoselova' ${ }^{1}$ G. M. Poletaev ${ }^{2, \dagger}$, V. V. Kovalenko ${ }^{3}$ \\ †gmpoletaev@mail.ru \\ ${ }^{1}$ Kuzbass Institute of the Federal Penitentiary Service of Russia, 49 Oktyabrsky str., Novokuznetsk, 654066, Russia \\ ${ }^{2}$ Altai State Technical University, 46 Lenin str., Barnaul, 656038, Russia \\ ${ }^{3}$ Siberian State Industrial University, 25 Bardin str., Novokuznetsk, 654007, Russia
}

\begin{abstract}
The effect of excess free volume on the mobility of triple junctions of grain boundaries by the example of nickel was studied by the molecular dynamics method. According to experimental data, triple junctions in polycrystals in most cases contain more specific free volume compared with the grain boundaries that form these junctions. Triple junctions of tilt boundaries with $<100>$ and $<111>$ misorientation axes were considered. Migration of the junctions was simulated by creating a nonequilibrium configuration of the joining boundaries - their migration, as a result of which they took an equilibrium position, led to migration of the triple junction (model proposed by L.S. Shvindlerman and co-authors). The interactions of nickel atoms with each other in the molecular dynamics model were described by the many-body Cleric-Rosato potential constructed within the tight binding model. The free volume was introduced locally into the triple junction region with the radius of $1 \mathrm{~nm}$. The percentage of the removed atoms from the considered region was varied from $0 \%$ to $80 \%$. Molecular dynamic simulation showed that the migration velocity of the triple junction is practically independent of the free volume value. This is explained by the fact that most part of the free volume introduced at the initial stage does not migrate with the triple junction but remains at the point where it was introduced and distributed along the boundary which lengthens during the migration of the junction. The smaller part of the free volume however remains in the joint but its presence has little effect on the mobility of the triple junction. This is mainly influenced by the mobility of the joining boundaries and the ratio of their tension.
\end{abstract}

Keywords: molecular dynamics, triple junction, grain boundary, migration, free volume.

УДК 538.911

\section{Исследование влияния избыточного свободного объема на подвижность тройных стыков границ зерен}

\author{
Новоселова Д. В. ${ }^{1}$, Полетаев Г. М., ${ }^{2 \dagger}$, Коваленко В. В. ${ }^{3}$ \\ ${ }^{1}$ Кузбасский институт ФСИН России, пр. Октябрьский, 49, Новокузнецк, 654066, Россия \\ ${ }^{2}$ Алтайский государственный технический университет им. И. И. Ползунова, пр. Ленина, 46, Барнаул, 656038, Россия \\ ${ }^{3}$ Сибирский государственный индустриальный университет, пр. Бардина, 25, Новокузнецк, 654007, Россия
}

\begin{abstract}
В настоящей работе проведено исследование методом молекулярной динамики влияния избыточного свободного объема на подвижность тройных стыков границ зерен на примере никеля. Согласно экспериментальным данным, тройные стыки в поликристаллах в большинстве случаев содержат больше удельного свободного объема по сравнению с образующими эти стыки границами зерен. Рассматривались тройные стыки границ наклона с осями разориентации $<100>$ и $<111>$. Миграция стыков моделировалась путем создания неравновесной конфигурации стыкующихся границ - их миграция, в результате которой они принимали равновесное положение, приводила к миграции тройного стыка (модель, предложенная Л. С. Швиндлерманом с соавторами). Взаимодействия атомов никеля друг с другом в молекулярно-динамической модели описывались многочастичным потенциалом Клери-Розато, построенным в рамках модели сильной связи. Свободный объем вводился локально в область тройного стыка радиусом 1 нм. Доля удаленных атомов из рассматриваемой области варьировалась от $0 \%$ до 80\%. Молекулярно-динамическое моделирование показало, что скорость миграции тройного стыка практически не зависит от величины свободного объема. Это объясняется тем, что большая часть введенного на начальном этапе свободного объема не мигрирует вместе с тройным стыком, а остается в том месте, где он был введен и распределяется вдоль границы, которая удлиняется в процессе миграции стыка. Меньшая часть свободного объема, тем не менее, остается в стыке, но его наличие слабо влияет на подвижность тройного стыка. На это преимущественно оказывают влияние подвижность стыкующихся границ и отношение их натяжений.
\end{abstract}

Ключевые слова: молекулярная динамика, тройной стык, граница зерен, миграция, свободный объем. 


\section{1. Введение}

Тройной стык границ зерен (или тройная линия) представляет собой линейный дефект, вдоль которого сопрягаются три различно ориентированных зерна или три зернограничных поверхности. Тройные стыки, как и границы зерен, могут мигрировать. Миграция тройных стыков и изменение их конфигурации играют важную роль при деформации поликристаллов с малым размером зерна, поэтому в настоящее время вопросу подвижности тройных стыков уделяется большое внимание. Классические теории роста зерна в обычных крупнозернистых поликристаллах, как правило, предполагают, что тройные стыки обладают бесконечной подвижностью, и рост зерна реализуется посредством миграции границ зерен. В рамках данных предположений кривизна границ зерен служит главной движущей силой для процесса роста зерен, причем тройные стыки границ зерен характеризуются равновесными углами во время такого процесса. Однако предположения, лежащие в основе классических теорий роста зерна, являются дискуссионными, особенно для случая нанокристаллических материалов [1]. Так, эксперименты [2-5] и результаты компьютерного моделирования роста зерен [6 -9] показали, что подвижность тройных стыков границ зерен является конечной величиной. В работах [2-9] отмечается, что существует два температурных участка движения системы границ с тройным стыком в металлах: при низких температурах стык оказывает тормозящее влияние на подвижность системы (стыковая кинетика) - его подвижность меньше, чем границ зерен; при высоких температурах подвижность системы определяется подвижностью границ зерен (граничная кинетика), то есть при высоких температурах стык становится более подвижным, чем границы. Для различных материалов и типов границ зерен существует различная температура перехода от стыковой к граничной кинетике.

Работы Л. С. Швиндлермана с соавторами [2 -9], выполненные с помощью прямых экспериментов и компьютерного моделирования, внесли существенный вклад в развитие представлений о подвижности тройных стыков границ зерен в металлах. Тем не менее, серьезные исследования в этом направлении начались сравнительно недавно, и в настоящее время остается ряд открытых вопросов. В первую очередь, это накопление численных данных, связанных со структурными и энергетическими характеристиками миграции тройных стыков для разных металлов в зависимости от различных внешних и внутренних факторов, исследование влияния на подвижность стыков примесей, дефектов кристаллической решетки (особенно вакансий и дислокаций), свободного объема. Кроме этого, важным вопросом является выяснение ведущих атомных механизмов процессов перестройки и миграции тройных стыков границ разного типа.

Настоящая работа посвящена исследованию методом молекулярной динамики влияния избыточного свободного объема на подвижность тройных стыков на примере никеля. Согласно экспериментальным данным, тройные стыки в поликристаллах в большинстве случаев содержат больше удельного свободного объема по сравнению с образующими эти стыки границами зерен. Диффузия в области тройного стыка границ зерен протекает значительно интенсивнее, чем вдоль самих границ [10], его структура является сравнительно более «рыхлой» [11], иногда даже с включениями аморфной фазы [12]. В работах $[13,14]$ с помощью компьютерного моделирования мы проанализировали различные причины формирования свободного объема в тройных стыках и пришли к выводу, что избыточный свободный объем образуется в стыках преимущественно в процессе кристаллизации в результате «запирания» плотности жидкой фазы при встрече трех фронтов кристаллизации и, как следствие, концентрирования избыточного свободного объема в тройном стыке после затвердевания.

\section{2. Описание модели}

Исследование проводилось с помощью программы MDR [15] на примере тройных стыков границ наклона с осями разориентации $<111>$ и $<100>$ в никеле. В настоящей работе за основу была взята конфигурация тройного стыка и модель, предложенная и развитая в работах Л. С. Швиндлермана с соавторами [2 - 9]. В отличие от работ, выполненных указанным коллективом с помощью компьютерного моделирования на примере двумерной модели $[7,8]$, нами было принято решение проводить исследования с использованием трехмерной модели. Относительно механизма миграции границ зерен, особенно малоугловых, двумерная и трехмерная модели имеют принципиальное отличие. В двумерной модели зернограничные краевые дислокации не имеют периодически расположенных вдоль ядер дислокаций изломов (ступенек), которые играют важную роль в зернограничных процессах: диффузии, миграции, деформации [16].

Расчетный блок создавался в виде пластины толщиной в 6 атомных плоскостей (Рис. 1). В случае стыка границ наклона $<111>$ расчетный блок имел высоту 25,9 нм, ширину 17,2 нм и толщину 1,2 нм. Для стыка границ $<100>$ размеры составляли 26,4 нм, 17,6 нм и 1,1 нм соответственно. Блоки содержали примерно 50000 атомов. Вдоль оси $Z$ (Рис. 1) имитировалось бесконечное повторение структуры, т.е. были наложены периодические граничные условия. По осям $X$ и $Y$ границы блока были жестко закреплены для фиксации заданной разориентации зерен. Это было сделано по двум причинам. Во-первых, границы зерен на краю расчетного блока должны быть зафиксированы, а это подразумевает сохранение ориентации кристаллической решетки трех разных зерен на границе блока в течение всего моделирования. Во-вторых, в расчетном блоке должно оставаться столько свободного объема, сколько было введено в начале, поскольку одной из задач исследования является изучение зависимости скорости миграции тройного стыка от величины свободного объема. 


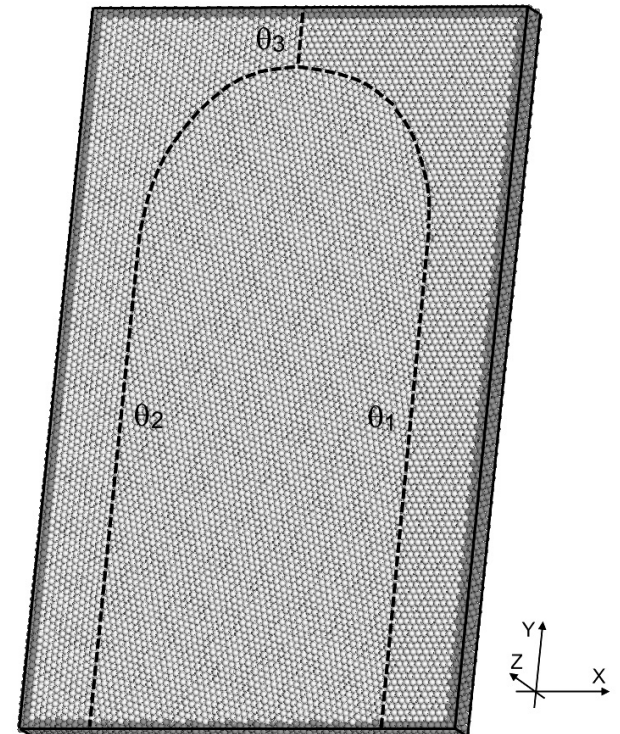

Рис. 1. Расчетный блок для моделирования миграции тройного стыка $<111>15^{\circ} / 15^{\circ} / 30^{\circ}$. Темно-серые атомы на краю расчетного блока в процессе компьютерного эксперимента оставались неподвижными (жесткие граничные условия). $\theta_{1}, \theta_{2}, \theta_{3}-$ углы разориентации границ зерен.

Fig. 1. Calculation block for modeling the migration of the $<111>15^{\circ} / 15^{\circ} / 30^{\circ}$ triple junction. Dark gray atoms on the edge of the calculation block remained motionless during the computer experiment (rigid boundary conditions). $\theta_{1}, \theta_{2}, \theta_{3}$ are the misorientation angles of the grain boundaries.

Согласно [2-9], рассматриваемая конфигурация границ (Рис. 1) должна способствовать постоянной (по крайней мере, на среднем участке) скорости миграции стыка в сторону противоположную оси Y. Сила, приводящая к миграции стыка, создается за счет натяжения границ 1 и 2, которые, натягиваясь, тянут вниз границу 3, в результате чего увеличивается ее длина, а длина границ 1 и 2 сокращается.

Рассматривались различные сочетания углов разориентации зерен, но большинство результатов было получено для стыков с углами разориентации $\theta_{1}=30^{\circ}, \theta_{2}=20^{\circ}$ и $\theta_{3}=10^{\circ}$ (в работе для краткости использовалось обозначение $<111>30^{\circ} / 20^{\circ} / 10^{\circ}$ или $<100>30^{\circ} / 20^{\circ} / 10^{\circ}$ в зависимости от направления оси разориентации границ наклона). Это было связано с высокой скоростью миграции данных стыков в модели, что облегчало исследование влияния на скорость миграции других факторов. Тройные стыки с углами разориентации зерен $15^{\circ} / 15^{\circ} / 30^{\circ}$, например, мигрировали в модели существенно медленнее, чем стыки $30^{\circ} / 20^{\circ} / 10^{\circ}$. При этом стыки границ $<100>$ при других равных условиях мигрировали медленней, чем стыки границ $\langle 111\rangle$. Первое, т.е. значительное отличие подвижности стыков $15^{\circ} / 15^{\circ} / 30^{\circ}$ и $30^{\circ} / 20^{\circ} / 10^{\circ}$, было вызвано двумя причинами. Во-первых, это было связано с отношением натяжений границ в стыке. Очевидно, что чем ниже натяжение границы 3, которая удлиняется в результате миграции стыка, по сравнению с натяжениями границ 1 и 2 (Рис. 1), тем быстрее должен мигрировать тройной стык. Во-вторых, на подвижность тройного стыка оказывает влияние подвижность самих границ. Известно, что малоугловые границы мигрируют медленнее большеуловых. Например, стыки $<111>5^{\circ} / 5^{\circ} / 10^{\circ}$ и $<100>5^{\circ} / 5^{\circ} / 10^{\circ}$ почти не сдвинулись с места при температуре $1700 \mathrm{~K}$ в течение сравнительно длительного по меркам молекулярной динамики моделирования - 4000 пс.

Взаимодействия атомов никеля друг с другом в молекулярно-динамической модели описывались многочастичным потенциалом Клери-Розато [17]. Данный потенциал хорошо зарекомендовал себя в ряде расчетов структурно-энергетических и термодинамических характеристик металлов, выполненных методом молекулярной динамики [18-21]. Шаг интегрирования по времени в методе молекулярной динамики был равен 5 фс. В $[22,23]$ было показано, что данного шага достаточно для моделирования тепловых движений атомов никеля вплоть до температуры плавления. Температура в модели задавалась через начальные скорости атомов согласно распределению Максвелла. Для сохранения температуры постоянной в процессе моделирования использовался термостат Нозе-Гувера.

\section{3. Результаты и обсуждение}

Прежде чем проводить исследование влияния свободного объема на скорость миграции тройного стыка, было проведено дополнительное исследование скорости миграции стыка в разные моменты времени в процессе его движения в компьютерной модели. Было выяснено, что скорость миграции не постоянна: на первом этапе происходит «натяжение» границ 1 и 2. Сам стык при этом практически остается на месте. После того, как границы 1 и 2 натягиваются, они начинают «тянуть» тройной стык и границу 3 вниз. В конце движения скорость постепенно падает из-за изменения направления векторов натяжений границ 1 и 2. В связи с этим скорость миграции тройного стыка должна измеряться только на среднем участке, когда движущая сила и углы между границами зерен в стыке постоянны.

Свободный объем вводился в цилиндрическую область радиусом 1 нм в районе тройного стыка путем удаления атомов (Рис. 2). Согласно нашим предыдущим исследованиям $[13,14,24]$, свободный объем концентрируется в тройном стыке при затвердевании в области, имеющей радиус около 1 нм. Доля удаленных атомов из рассматриваемой области варьировалась от $0 \%$ до 80\%. После введения свободного объема (удаления атомов) проводилась структурная релаксация при начальной температуре $0 \mathrm{~K}$, в течение которой свободный объем распределялся в области стыка.

Измерение скорости миграции проводилось при температуре 1700 К. При этой температуре, близкой к температуре плавления никеля, миграция стыка происходила с достаточно высокой скоростью, чтобы ее можно было измерять в молекулярно-динамической модели. Графики зависимостей скорости миграции от доли удаленных атомов в области радиусом 1 нм для стыков $<111>30^{\circ} / 20^{\circ} / 10^{\circ}$ и $<100>30^{\circ} / 20^{\circ} / 10^{\circ}$ приведены на Рис. 3. 
Как видно из Рис. 3, скорость миграции рассматриваемых стыков в результате введения избыточного свободного объема почти не изменилась. Даже при сравнении скорости при $0 \%$ и $60 \%$ разница совсем небольшая, в пределах погрешности: 11,4 и 12,3 м/с для стыка $<111>30^{\circ} / 20^{\circ} / 10^{\circ}$ и 3,6 и $3,9 \mathrm{м} /$ с для $<100>30^{\circ} / 20^{\circ} / 10^{\circ}$ при $1700 \mathrm{~K}$.

При наблюдении за распределением свободного объема в расчетном блоке в процессе миграции стыка, стало понятна причина отсутствия его влияния на скорость миграции стыка. На Рис. 4 изображены распре-

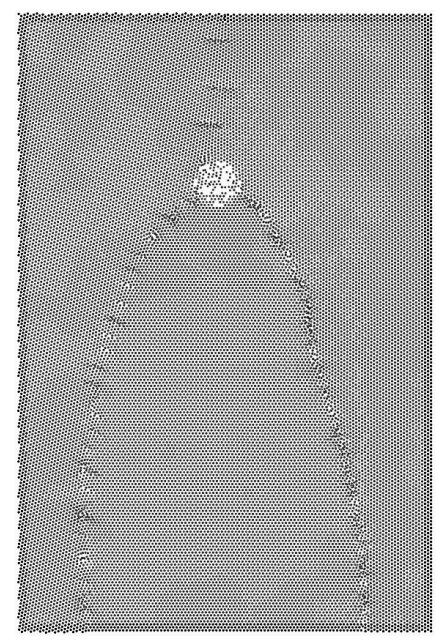

a

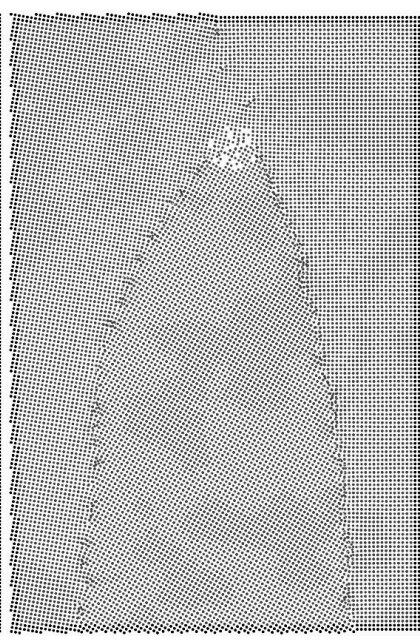

b
Рис. 2. Введение свободного объема в область тройного стыка в начале компьютерного эксперимента (путем удаления $60 \%$ атомов в области радиусом $1 \mathrm{HM}$ ): $<111>30^{\circ} / 20^{\circ} / 10^{\circ}$ (a), $<100>30^{\circ} / 20^{\circ} / 10^{\circ}(\mathrm{b})$

Fig. 2. Introduction of the free volume into the triple junction region at the beginning of the computer experiment (by removing 60\% of atoms in the region of radius $1 \mathrm{~nm}$ ): $<111>30^{\circ} / 20^{\circ} / 10^{\circ}$ (a), b) $<100>30^{\circ} / 20^{\circ} / 10^{\circ}$ (b).

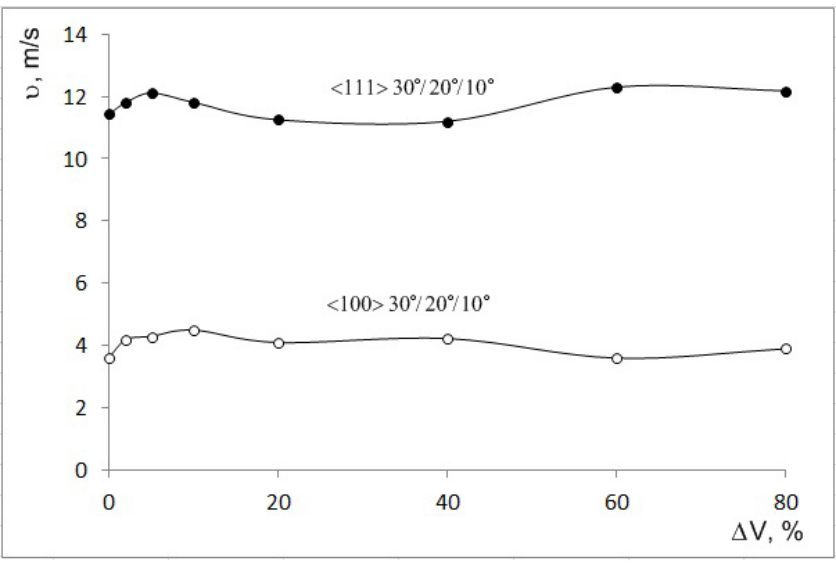

Рис. 3. Графики зависимостей скорости миграции от доли удаленных атомов в области радиусом 1 нм для стыков $<111>30^{\circ} / 20^{\circ} / 10^{\circ}$ и $<100>30^{\circ} / 20^{\circ} / 10^{\circ}$ при температуре $1700 \mathrm{~K}$.

Fig. 3. Graphs of the dependence of the migration velocity on the percentage of removed atoms in the region of radius $1 \mathrm{~nm}$ for $\langle 111\rangle 30^{\circ} / 20^{\circ} / 10^{\circ}$ and $\langle 100\rangle 30^{\circ} / 20^{\circ} / 10^{\circ}$ junctions at the temperature of $1700 \mathrm{~K}$. деления свободного объема с помощью визуализатора средних расстояний между соседними атомами в расчетных блоках после моделирования миграции рассматриваемых стыков. Визуализация свободного объема осуществлялась путем расчета среднего расстояния от каждого атома до ближайших атомов. Если среднее расстояние незначительно отличалось от расстояния, соответствующего идеальному кристаллу, атом не изображался. В противном случае атом закрашивался в тот или иной оттенок серого цвета.

По приведенным рисункам видно, что введенный свободный объем не следует за мигрирующим стыком, а остается в том месте, где был введен и распределяется вдоль границы, которая удлиняется в процессе миграции стыка (т. е. границы 3). Часть свободного объема все же, как видно из рисунков, остается в стыке, но это практически не влияет на скорость миграции. Дело, видимо, в том, что снизу стыка, на пути миграции, избыточного свободного объема нет, тогда как именно наличие его по ходу движения, в большей степени должно оказывать влияние на скорость миграции. Однако введение избыточных вакансий по ходу движения стыка и исследование изменения скорости миграции стыка в этом случае является другой задачей, которая в настоящей работе не рассматривалась, как и не рассматривалось, например, влияние на миграцию стыка примесей и решеточных дислокаций.

Таким образом, часть избыточного свободного объема в процессе миграции тройного стыка остается в начальной позиции стыка, распределяясь преимущественно в границе зерен, которая удлиняется в результате

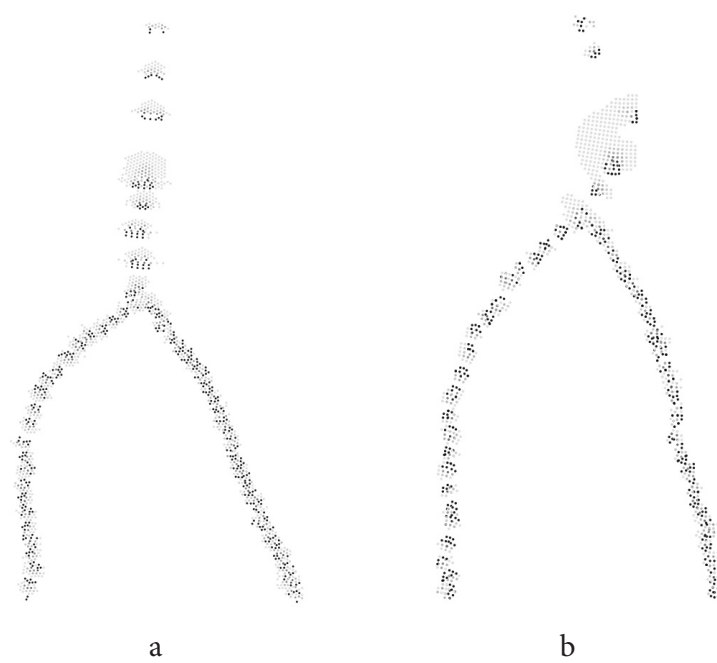

Рис. 4. Распределение свободного объема в расчетных блоках, содержащих тройнойные стыки <111> 30\%/20\% $10^{\circ}$ (а) и $<100>30^{\circ} / 20^{\circ} / 10^{\circ}$ (b), после компьютерного эксперимента в течение 500 пс при температуре 1700 К. Было удалено 60\% атомов в области стыка радиусом 1 нм в начале компьютерного эксперимента.

Fig. 4. Distribution of the free volume in the calculation blocks containing $<111>30^{\circ} / 20^{\circ} / 10^{\circ}$ (а) и $<100>30^{\circ} / 20^{\circ} / 10^{\circ}$ (b) triple junctions after the computer experiment for 500 ps at temperature of $1700 \mathrm{~K} .60 \%$ of atoms were removed in the junction region of radius $1 \mathrm{~nm}$ at the beginning of the computer experiment. 
миграции. В тройном стыке устанавливается равновесное содержание свободного объема, при этом величина свободного объема в самом стыке слабо влияет на его подвижность. На это оказывают влияние преимущественно подвижность стыкующихся границ и отношение их натяжений.

\section{4. Заключение}

В настоящей работе проведено исследование методом молекулярной динамики влияния избыточного свободного объема на подвижность тройных стыков на примере никеля. Свободный объем вводился локально в область тройного стыка радиусом 1 нм. Рассматривались тройные стыки границ наклона с осями разориентации $<100>$ и $<111>$. Показано, что скорость миграции тройного стыка практически не зависит от величины свободного объема. Это объясняется тем, что большая часть введенного на начальном этапе свободного объема не мигрирует вместе с тройным стыком, а остается в том месте, где он был введен и распределяется вдоль границы, которая удлиняется в процессе миграции стыка. Меньшая часть свободного объема, тем не менее, остается в стыке, но его наличие слабо влияет на подвижность тройного стыка. На это преимущественно оказывают влияние подвижность стыкующихся границ и отношение их натяжений.

Благодарность/Acknowledgements. Исследование выполнено при финансовой поддержке Министерства образования и науки РФ в рамках базовой части государственного задания (проект № 3.4820.2017/БЧ) и гранта РФФИ № 16-48-190182 p_a./The reported study was partially supported by Ministry of Education and Science of the Russian Federation within the framework of the state task (project No. 3.4820.2017/8.9) and RFBR (project No. 16-48-190182-r_a).

\section{Литература/References}

1. I. A. Ovid'ko. Materials Physics and Mechanics. 8 (2), 174 (2009).

2. G. Gottstein, V. Sursaeva, L. Shvindlerman. Interface Science. 7, 273 (1999).

3. S. G. Protasova, V.G. Sursaeva, L.S. Shvindlerman. Physics of the Solid State. 45, 1471 (2003).

4. U. Czubayko, G. Gottstein, V.G. Sursaeva, L. S. Shvindlerman. Acta Materialia. 46 (16), 5863 (1998).

5. G. Gottstein, L. S. Shvindlerman, V. G. Sursaeva. Scripta Materialia. 116, 91 (2016).

6. G. Gottstein, Y. Ma, L.S. Shvindlerman. Acta Materialia. 53, 1535 (2005).

7. M. Upmanyu, D. J. Srolovitz, L.S. Shvindlerman, G. Gottstein. Interface Science. 7, 307 (1999).

8. M. Upmanyu, D. J. Srolovitz, L.S. Shvindlerman, G. Gottstein. Acta Materialia. 50, 1405 (2002).

9. C. Mießen, M. Liesenjohann, L.A. Barrales-Mora, L. S. Shvindlerman, G. Gottstein. Acta Materialia. 99, 39 (2015).

10. G. Palumbo, K. T. Aust. Scripta Metallurgica et Materialia.
24, 1771 (1990).

11. A. I. Gusev. Physics-Uspekhi. 41, 49 (1998).

12. P. Rodriguez, D. Sundararaman, R. Divakar, V.S. Raghunathan. Chemistry for Sustainable Development. 8, 69 (2000).

13. G. M. Poletaev, D. V. Novoselova, V.M. Kaygorodova, M.D. Starostenkov. Fundamentalnye problemy sovremennogo materialovedenia. 12 (2), 253 (2015) (in Russian) [Г.. Полетаев, Д.В. Новоселова, B.M. Кайгородова, М.Д. Старостенков Фундаментальные проблемы современного материаловедения. 12 (2), 253 (2015)].

14. G. M. Poletaev, D. V. Novoselova, V. M. Kaygorodova. Solid State Phenomena. 249, 3 (2016).

15. Полетаев Г.M. Molecular dynamics research (MDR). Свидетельство о государственной регистрации программы для ЭВМ № 2015661912 от 12.11.2015.

16. G. M. Poletaev, A.B. Yuryev, V.E. Gromov, M.D. Starostenkov. Atomic mechanisms of structuralenergy transformations near tilt grain boundaries in fcc metals and $\mathrm{Ni}_{3} \mathrm{Al}$ intermetallide. Novokuznetsk, SibSIU. (2008) 160 р. [Г. М. Полетаев, А. Б. Юрьев, В. Е. Громов, М.Д. Старостенков. Атомные механизмы структурно-энергетических превращений вблизи границ зерен наклона в ГЦК металлах и интерметаллиде $\mathrm{Ni}_{3}$ Al. Новокузнецк, СибГИУ. (2008) 160 c].

17. F. Cleri, V. Rosato. Physical Review B. 48 (1), 22 (1993).

18. G. M. Poletaev, D.V. Dmitrienko, V.V. Diabdenkov, V.R. Mikrukov, M.D. Starostenkov. Physics of the Solid State. 55 (9), 1920 (2013).

19. G. M. Poletaev, M.D. Starostenkov, S. V. Dmitriev. Materials Physics and Mechanics. 27 (1), 53 (2016).

20. G. M. Poletaev, M.D. Starostenkov. Technical Physics Letters. 35 (1), 1 (2009).

21. R.Yu. Rakitin, G.M. Poletaev, M.S. Aksenov, M. D. Starostenkov. Technical Physics Letters. 31 (8), 650 (2005).

22. A. A. Valuev, G.E. Normann, V.Y. Podlipchuk. Method of molecular dynamics: theory and applications/In: Mathematical modeling: Physicochemical properties of matter. Moscow, Nauka. (1989) p. 5-40 [А.А. Валуев, Г.Э. Норманн, В.Ю. Подлипчук. Метод молекулярной динамики: теория и приложения/В кн.: Математическое моделирование: Физикохимические свойства вещества. Москва, Наука (1989) C. $5-40]$.

23. M.D. Starostenkov, N.N. Medvedev, G.M. Poletaev. To the question of systematic errors in $\mathrm{MMD} / \mathrm{In}$ : Measurements, automation and modeling in industry and scientific research (Ed.G. V. Leonov). Barnaul, AltSTU (2005) p. 5-8 [М.Д. Старостенков, Н.Н. Медведев, Г.М. Полетаев. К вопросу о систематических погрешностях в ММД/В кн.: Измерения, автоматизация и моделирование в промышленности и научных исследованиях (под. ред. Г. В. Леонова). Барнаул, АлтГТУ (2005) C. 5 -8].

24. G. M. Poletaev, D. V. Novoselova, I. V. Zorya, M. D. Starostenkov. Materials Physics and Mechanics. 30 (1), 68 (2017). 